Canadian Oncology

Nursing Journal

Revue canadienne

de soins infirmiers

en oncologie

Volume 30, Issue 4 • Fall 2020

elSSN: 2368-8076 


\title{
Optimizing the delivery of early palliative care for hematology patients receiving a stem cell transplant: A role for a Nurse Practitioner
}

\author{
by Ellen A. Lewis
}

\begin{abstract}
A hematopoietic stem cell transplant (HSCT) is an intense treatment approach for patients with a hematologic malignancy and brings a significant risk for morbidity and mortality. HSCT brings hope of cure for patients; however, treatments are lengthy and burdensome from both a physical and psychosocial perspective. As the culture of HSCT has traditionally been cure-oriented, it leaves little room for a potential partnership with palliative care services, and when palliative care services are introduced, it is often too late for significant benefit. The need to standardize palliative care involvement for patients with hematologic malignancies receiving a HSCT has become necessary, yet there are challenges with the best way to create such a partnership. Nurse practitioners are a steadily growing professional body possessing the knowledge and skill necessary to fill the gap in palliative care delivery for HSCT patients. The proposed Nurse Practitioner Early Palliative Care for HSCT patients (NEST) algorithm will seek to provide a pathway in which to foster a partnership between hematology/HSCT teams and palliative services to yield the best care possible for patients.
\end{abstract}

Key words: quality of life, hematopoietic stem cell transplant, palliative care, early palliative care, nurse practitioner

\section{INTRODUCTION}

$C$ oncurrent delivery of palliative care services for oncology patients has been advocated for in recent literature due to its benefits, which include improving quality of life, increasing overall survival, and reducing healthcare costs (El-Jawarhi et al., 2016; Ferrell et al., 2017; May et al., 2017; Temel et al., 2010). Recently, authors have highlighted the important role that nurse practitioners (NPs) can play in providing palliative care to oncology patients (Dyar et al., 2012; Mitchell et al., 2016; Owens et al., 2012).

Despite the documented benefits, palliative care for hematology patients, especially those receiving a hematopoietic stem-cell transplant (HSCT), presents a particular problem as the unpredictable illness trajectory can make it

\section{AUTHOR NOTE}

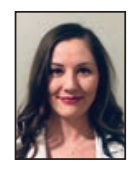

Ellen A. Lewis, RN, BN, MN, NP, Nurse Practitioner, Hematology, Tom Baker Cancer Centre, Foothills Medical Centre, $\mathrm{AB}$

DOI:10.5737/23688076304239245 a challenge to know when to involve specialized palliative care services (Levine et al., 2017). Although the Canadian Medical Association (CMA), the National Comprehensive Cancer Network (NCCN), and the American Society of Clinical Oncology (ASCO) have endorsed palliative care for its benefits in oncology care, the integration of early palliative care (EPC) is rarely systematically delivered and this disparity is even more apparent for hematology patients (CMA, 2015; Dans et al., 2017; El-Jawahri et al., 2016; Ferrell et al., 2017).

In this paper, literature regarding the importance of EPC for hematology and HSCT patients and the benefits of palliative care NPs will be presented. A clinical pathway (Figure 1) depicting how palliative care NPs can be integrated into the standard care of HSCT patients will be proposed.

\section{BACKGROUND}

A HSCT is often one of the only treatment options aimed at gaining long-term control or cure for patients with a hematologic malignancy. Hematopoietic stem-cell transplant is an aggressive treatment approach and, as such, can be accompanied by organ toxicities, graft failure, substantial symptom burden, poor quality of life, and significant risk of mortality (Chung et al., 2009; El-Jawahri et al., 2015). Although the outcomes after a HSCT are improving, a chance of disease cure often cannot be attained without prolonged aggressive chemotherapy and risk of death from infection or organ failure (D'souza et al., 2017; Levine et al., 2017). If treatments are not successful, patients are often subject to aggressive end of life care involving intensive care admissions and salvage chemotherapy within the last weeks of their lives (Ho et al., 2011; Hui et al., 2014). Given the unpredictable illness trajectory and the challenge of knowing when to involve specialized palliative care services, hematology patients and families can be unprepared when end of life or significant disease morbidity presents (Levine et al., 2017).

Most recently, authors have proposed the need for concurrent delivery of palliative care services alongside curative care for patients receiving a HSCT (El-Jawahri et al., 2016; Harden \& Schembri, 2016; Loggers et al., 2016; Roeland \& Ku, 2015; Selvaggi et al., 2014). In addition, ASCO has developed guidelines recommending the integration of palliative care services within eight-weeks of diagnosis for patients with difficult to control physical or psychosocial symptom or those with advanced cancer (defined as distant metastasis, late-stage disease, prognosis of 6-24 months, or life-limiting malignancy) 


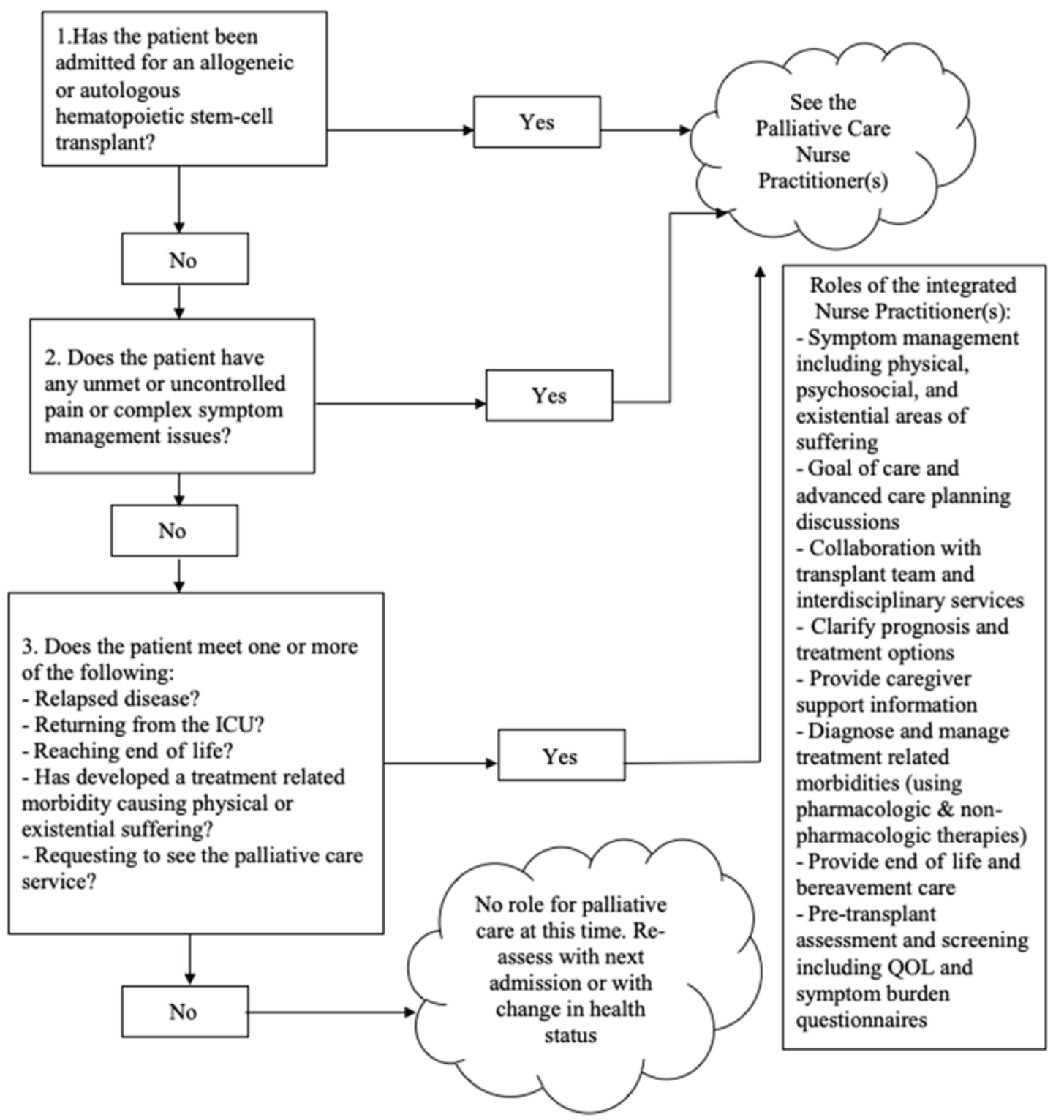

Figure 1: NEST Pathway

(Ferrell et al., 2017; Smith et al., 2012). NPs are cited as effective palliative care providers in recent pilot projects, making them an optimal choice to help facilitate EPC for HSCT patients (Dyar et al., 2012; Mitchell et al., 2016; Owens et al., 2012).

Specialized palliative care services consist of team members with focussed training aimed at promoting and supporting the quality of life of patients and their caregivers. The palliative care service is responsible for working with patients to ensure a clear understanding of their disease, clarify prognosis and treatment goals, support both physical and existential symptom needs, assist with decision-making, and help understand and elucidate patient's wishes (Ferrell et al., 2017). Unfortunately, the palliative care service is often only associated with end of life care and, until recently, has not been viewed as a service that could be complementary to curative care.

\section{LITERATURE REVIEW}

A systematic search was conducted of published literature regarding NPs, EPC, and oncology using the Cumulative Index of Nursing and Allied Health Literature (CINAHL), MEDLINE, PubMed, and Google Scholar databases. The following search terms were used: palliative care, early palliative care, end of life care, and supportive care in conjunction with cancer, malignancy, neoplasm, oncology, bone marrow transplant, stem cell transplant, hematopoietic transplant, hematology, and advanced cancer. Additional searches pertaining to NPs were conducted using the search terms: nurse practitioner and advanced practice nurse in conjunction with the above oncology and palliative care search terms. The search was restricted to English language publications from 1990 onward. Reference lists of included articles were checked to ensure all relevant literature was included. 


\section{The Current State of Early Palliative Care}

The introduction of EPC services for cancer patients has the potential of improving quality of life and overall survival, decreasing inappropriate aggressive end of life care interventions, and reducing healthcare costs (Bakitas et al., 2009; Ho et al., 2011; May et al., 2017; Temel et al., 2010; Temel et al., 2011; Zimmermann et al., 2014). In 2010, Temel and associates published a single-site randomized controlled trial (RCT) with 151 metastatic non-small cell lung cancer patients and compared the effect of an EPC intervention $(n=77)$ versus standard oncology care $(n=74)$. Temel et al. (2010) discovered a statistically significant improvement in quality of life for the EPC patients measured by three separate scales: The Functional Assessment of Cancer Scale $(\mathrm{p}=.03)$, the Lung Cancer Subscale $(\mathrm{p}=.04)$, and the Trial Outcome Index $(\mathrm{p}=.04)$. Additional findings included a statistically significant overall longer survival, 11.6 versus 8.9 months $(\mathrm{p}=.02)$, and less aggressive end of life care (54\% [ $n=56]$ vs $33 \%$ [ $n=49] ; \mathrm{p}=.05)$ favouring the EPC group. In a separate publication, Temel et al., (2011) found that the EPC group developed a more accurate perception of their prognosis compared to standard care group $(82.5 \%[n=57]$ vs $59.6 \%[n=47] ; \mathrm{p}=.02$ ). The benefits of EPC documented by Temel et al. (2010 \& 2011) subsequently inspired multiple other trials studying the effects of EPC (Bakitas et al., 2015; May et al., 2017; Zimmerman et al., 2014).

Zimmermann et al. (2014) conducted an RCT with 461 solid-tumour oncology patients again randomized to receive an EPC intervention $(n=228)$ versus standard oncology care $(n=233)$. A statistically significant difference in quality of life at three months $(\mathrm{p}=.05)$ and at four months $(\mathrm{p}=.003)$ was found, favouring the EPC group (Zimmermann et al., 2014). The ENABLE III RCT conducted by Bakitas and associates (2015) with 207 advanced cancer patients randomized to receive EPC (within one-two months after diagnosis; $n=104$ ) and delayed palliative care (three or more months after diagnosis; $n=103$ ) noted a statistically significant increase in overall survival after one year amongst those patients who had died by the end of data collection $(n=109)$, favouring the EPC group (63\% [ $n=59]$ versus $48 \%$ [ $n=50] ; p=.038)$. Additional benefits of EPC were demonstrated in a multisite prospective cohort study conducted by May et al. (2017), which consisted of 863 advanced cancer patients who received usual care $(n=637)$, early palliative care (within two days of admission; $n=177$ ), or delayed palliative care (more than two days post-admission; $n=49$ ). May et al. (2017) noted a cost-saving benefit ( $\$ 1539$ USD) per hospitalization attributable to decreased length of stay (\$968 USD [63\%]) and reduced intensity of treatments (\$571 USD [37\%]) favouring the EPC cohort.

Although the large majority of research regarding EPC has involved solid-tumour oncology patients, it can be posited that hematology and HSCT patients would also benefit from EPC involvement (Loggers et al., 2016). In a multisite cross-sectional analysis consisting of 1,235 cancer patients, Hochman et al. (2018), compared the difference in palliative care symptom needs between hematology patients $(n=108)$ and the symptom needs of solid-tumour oncology patients $(n=1127)$. Hochman et al. (2018) documented no statistically significant difference between the groups in the areas of pain, anxiety, dyspnea, constipation, nausea, depression, appetite, and well-being, although hematology patients had statistically significant greater odds of being tired (odds ratio [OR] 2.19; 95\% confidence interval [CI] 1.22-3.91; $\mathrm{p}<.01$ ) and drowsy (OR 1.81; 95\% CI 1.07-3.07; $\mathrm{p}<.03)$ compared to the solid tumour oncology group (Hochman et al., 2018).

Despite similar symptom needs, it is perplexing to note that the referral rates to palliative care services are much lower for hematology patients. A meta-analysis conducted by Howell et al. (2010), noted that hematologic patients were less likely to obtain care from a specialized palliative care service when compared to solid-tumour oncology patients (risk ratio 0.46 ; 95\% CI 0.42-0.50). Additionally, a mixed method study conducted by LeBlanc et al. (2015) comparing differences in referral patterns to palliative care service between hematologists $(n=23)$ and oncologists $(n=43)$ discovered that hematologists were more likely to associate palliative care with end of life care $(61 \%[n=14]$ versus $16 \%,[n=6] ; \mathrm{p}=.001)$, and $30 \%$ $(n=7)$ of hematologists had never referred a patient to palliative care $(\mathrm{p}=.001)$. In summary, the overwhelming intensity of a HSCT, the likelihood of receiving aggressive end of life care, and the lack of palliative care referrals all exhibit a strong need to standardize EPC for patients with a hematologic malignancy. Accordingly, standardized EPC has the potential to yield improved quality of life for patients.

\section{Early Palliative Care in HSCT Patients}

El-Jawahri et al. (2015) provided the two most rigorous studies regarding symptom burden of HSCT and the need for EPC. In the first publication, El-Jawahri et al. (2015) offered a longitudinal cohort design that studied the impact HSCT had on the quality of life of hematologic patients (autologous $n=30$; reduced intensity allogenic $n=30$; myeloablative allogenic $n=30)$ and their caregivers $(n=47)$. In regard to quality of life, El-Jawahri et al. (2015) demonstrated that the percentage of patients with clinically significant depressive symptoms doubled from baseline to day eight post HSCT $(15.6 \%$ to $37.8 \%$; $n=72 ; \mathrm{p}=.005)$ and the percentage of patients who would meet the diagnostic criteria for major depression increased (7.8\% to $36.7 \% ; n=72 ; \mathrm{p}=.0001$ ). Additionally, patients' quality of life scores, measured on the Functional Assessment of Cancer Therapy-Bone Marrow Transplant Index, decreased from 109.6 to 96.0 ( $\mathrm{p}<.0001$; El-Jawahri et al., 2015). The caregiver results showed a statistically significant decline in quality of life $(\mathrm{p}=.03)$, physical functioning $(\mathrm{p}=.02)$, general health $(\mathrm{p}=.0007)$, vitality $(\mathrm{p}=.003)$, social functioning $(\mathrm{p}=.004)$, emotional functioning $(\mathrm{p}=.05)$, and an increase in depression scores $(\mathrm{p}=.03$; El-Jawhari et al., 2015). El-Jawahri et al. (2015) asserted that hematologic patients undergoing a HSCT and their caregivers have a substantial need for supportive care interventions during their inpatient admission.

In a more recent RCT, El-Jawahri et al. (2016) provided evidence for EPC integration into standard care for hematology patients undergoing a HSCT by randomizing 160 hematology patients and 94 caregivers to receive EPC intervention (patients $n=81$; caregivers $n=49$ ) versus standard care 
(patients $n=79$; caregivers $n=45$ ). At two weeks post HSCT, statistically significant improvements in patients' quality of life ( $\mathrm{p}=.02 ; 95 \%$ CI 1.27 to 14.19$)$, anxiety ( $\mathrm{p}<.001 ; 95 \%$ CI -3.22 to -1.29$)$, and symptom burden ( $\mathrm{p}=.02 ; 95 \% \mathrm{CI}-11.46$ to -1.05$)$ were noted, favoring the EPC group (El-Jawahri et al., 2016). At three months, statistically significant improvements were seen in the EPC patients' post-traumatic stress disorder scores ( $\mathrm{p}=.002$; 95\% CI -7.12 to -1.58; El- Jawahri et al., 2016). Despite these benefits presented in the literature, routine involvement of palliative care services is not standard care for the majority of transplant centres, especially those in Canada.

\section{BARRIERS AND FACILITATORS TO EARLY PALLIATIVE CARE IMPLEMENTATION}

There are many barriers preventing the successful implementation of EPC for HSCT patients including the stigma associated with palliative care, unpredictability of hematologic malignancies, difference between oncologist and palliative care philosophies, and misunderstanding of the appropriate timing for EPC (Kain \& Eisenhauer, 2016; Pfeil et al., 2015; Roeland \& Ku, 2015). Hematologists more often view palliative care as only being useful in end of life and, therefore, might be less inclined to consult the service (LeBlanc et al., 2015). Additionally, palliative care services are not thought to have a role in the curative HSCT approach and are associated with being a "death squad" (Chung et al., 2009, p. 265; Levine et al., 2017). The culture of hematology and HSCT have been cure-oriented traditionally and have not been viewed as aligning with the culture of palliative care services. The challenge in the future will be to shift conceptions and rebuild the structure on which to support hematologic patients and those receiving a HSCT.

It is necessary to find creative ways to mitigate barriers preventing EPC and facilitate a partnership between HSCT teams and palliative care services, in hopes of optimizing EPC delivery. One way would be to identify health professions who can contribute to the successful implementation of EPC (Dyar et al., 2012; Owens et al., 2012). NPs are cost-effective and autonomous professionals who encompass strong clinical and collaborative skills making them well positioned to fill this gap and provide EPC to HSCT patients (Bakitas et al., 2015; Canadian Nurses Association, 2018; Deitrick et al., 2011; Fox, 2014; Kennedy et al., 2015; Walling et al., 2017). The addition of trained palliative care NPs working exclusively as a part of a hematology/HSCT team in an integrated role could influence the uptake of EPC for the hematology and HSCT patient populations.

\section{NURSE PRACTITIONER DELIVERED PALLIATIVE CARE}

Authors have reported that NPs are effective in supporting the palliative care needs of oncology patients (Bakitas et al., 2015; Clark et al., 2017; Dyar et al., 2012; Mitchell et al., 2016; Owens et al., 2012). For instance, Dyar et al. (2012) randomized patients to receive an NP directed supportive care intervention $(n=12)$ versus standard care alone $(n=14)$, and found a statistically significant improvement in patients' emotional quality of life $(\mathrm{p}=.0106)$ and in the mental quality of life $(p=.0219)$ favouring the intervention group. Additionally, integrated palliative care NP roles can reduce emergency department visits, lead to more advanced care planning documentation, and increase referrals to hospice and psychosocial supports (Owens et al., 2012; Walling et al., 2017). Walling et al. (2017) conducted a prospective cohort study that evaluated the impact that an NP-led palliative care intervention $(n=224)$ had on outpatient breast cancer patients, compared to standard care alone $(n=2370)$. Wallings et al. (2017) demonstrated that patients in the intervention group were more likely to have advanced care planning documents $(30 \%$ [ $n=224]$ versus $17 \%$ $[n=2370] ; \mathrm{p}<.01)$, receive a referral to psychosocial support $(46 \%[n=224]$ versus $16 \%[\mathrm{n}=2370] ; \mathrm{p}<.01)$, and receive a hospice referral $(53 \%[n=224]$ versus $23 \%[n=2370] ; \mathrm{p}=.02)$ when compared to the standard care group (Walling, et al., 2017). Furthermore, Owens et al. (2012) published a pilot project studying the impact an NP-led outpatient palliative care service had on cancer patients $(n=49)$ and it was noted that the number of emergency department visits decreased from 0.07 visits per week in 2009 to 0.04 visits per week in 2010 ( $p=.001$ ) demonstrating that NP-led palliative care intervention decreased emergency department visits (Owens et al., 2012).

\section{CLINICAL PATHWAY DEVELOPMENT}

As EPC has demonstrated its benefit for oncology patients, the primary issue, currently, is how to optimize its delivery. Given the evidence, NPs are well-positioned to address the quality of life and holistic needs that accompany a diagnosis of advanced cancer and the complications that accompany a HSCT. Unfortunately, it has been demonstrated that patients with a hematologic malignancy are not likely to receive input from specialized palliative care services during their admission for HSCT, emphasizing a need for the development of a clinical pathway depicting how HSCT patients and palliative care services will align (LeBlanc et al., 2015; Harden \& Schembri et al., 2016; Howell et al., 2010).

\section{NEST Pathway: Nurse-Practitioner-Delivered Early Palliative

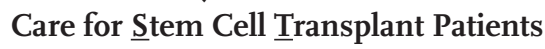

The NEST clinical pathway (Figure 1) depicts how a palliative care specialized NP can be directly integrated as a part of a hematology/HSCT unit(s) and provide EPC to patients with a hematologic malignancy who are receiving a HSCT. Universal palliative care support will be provided to every HSCT patient by the palliative care $\mathrm{NP}(\mathrm{s})$. Additionally, palliative care support will be provided by the NP(s) if hematology patients have unmet or uncontrolled symptoms or are displaying indicators of poor quality of life prior to their HSCT admission. The role of EPC involvement would be assessed at every admission and more frequently if necessary.

After a review of the literature, the NEST Pathway appears to represent the first clinical pathway developed specifically addressing the delivery of EPC for adult hematology/HSCT inpatients. Use of this pathway could influence the traditional cure-based culture of HSCT and hematology and forge a new 
frontier for transplant care. Centres that implement the pathway could be seen as leaders in the hematology/HSCT world and influence the uptake of EPC for patients.

\section{Facilitators to Integrating the NEST Pathway}

The NEST Pathway provides an algorithm to combat the lack of EPC involvement for HSCT patients. This clinical pathway allows for every HSCT patient to be seen by an integrated palliative care NP, so patients and their families feel well prepared if cure is unattainable, or when end of life or disease morbidity presents. This pathway permits the NP to place the symptom burden, mitigation of suffering, and quality of life at the foreground, allowing for HSCT teams to focus on treatment goals and longer-term morbidity and mortality prevention. Restructuring the delivery of care in this way fosters a clinical partnership between palliative care services and HSCT teams to provide comprehensive care to patients (Levine et al., 2017).

Given the unpredictable nature of hematologic malignancies, indicators that a patient may require a palliative care consult may not always be obvious until it is too late for significant palliative care benefit. American Society of Clinical Oncology guidelines highlight indicators such as frequent admissions, difficult-to-control symptoms, complex management, including antibiotics and feeding support, and a decline in functioning as reasons to make a palliative care referral (Ferrell et al., 2017). A large majority of HSCT patients and many hematology patients should obtain a palliative care referral based on these indicators alone. However, referral rates continue to be low (Howell et al., 2010; LeBlanc et al., 2015). When the NEST Pathway becomes part of the "standard of care" for these patients, it allows these patients to receive the added benefits of EPC support.

The NEST Pathway employs the help of NPs, who are highly skilled, knowledgeable, cost-effective, and excel at interdisciplinary collaboration. The integration of a palliative care NP would encourage the utilization of the profession to its full scope of practice and advocate for more NP jobs in cancer care areas. Furthermore, the NEST Pathway could decrease provider stress and burnout by encouraging shared care and providing the opportunity between team members to make decisions regarding challenging patient situations (D'Ambruso et al., 2016).

\section{Barriers to Integrating the NEST Pathway}

A downside to the NEST universal palliative care pathway is that it could be more resource-intensive when compared to traditional trigger-based models, based on referrals happening only when patients meet certain indicators (Levine et al., 2017).

\section{REFERENCES}

Bakitas, M., Lyons, K. D., Hegel, M. T., Balan, S., Brokaw, F. C., Seville, J., Hull, J. G., Li, Z., Tosteson, T. D., Byock, I. R., \& Ahles, T. A. (2009). Effects of a palliative care intervention on clinical outcomes in patients with advanced cancer: The project ENABLE II randomized controlled trial. JAMA, 302(13), 741-749. https://doi. org/10.1001/jama.2009.1198

Bakitas, M. A., Tosteson, T. D., Li, Z., Lyons, K. D., Hull, J. G., Li, Z., Dionne-Odom, J. N., Frost, J., Dragnev, K. H., Hegel, M. T., Azuero, A., Ahles, T. A. (2015). Early versus delayed initiation of concurrent
Additionally, depending on the size of the transplant centre and the acuity of the patients, multiple NPs may need to be employed. However, NPs are cost-effective providers, and this barrier could become less burdensome (O'Mahony et al., 2017). The use of palliative care NPs in other areas, such as the intensive care unit, has shown cost savings benefits. After integrating palliative care NPs, O'Mahony et al. (2017) accounted a cost savings of $\$ 224,500$ USD over a six-month period from shorter hospital stays and preventing unnecessary hospital testing.

An additional barrier to the NEST Pathways could be that traditional physician-led HSCT teams would have to be willing to integrate a palliative care NP. This barrier could be mitigated by emphasizing the shared responsibility of patient care. As the needs of cancer patients are complex, it is unrealistic to expect all the physical, psychosocial, existential, and spiritual needs of patients should be handled by the hematology or HSCT team alone (D'Ambruso et al., 2016). Allowing for sub-specialties to focus within their level of expertise will save time and would yield higher-quality care.

\section{RECOMMENDATIONS FOR FUTURE RESEARCH}

Future research should focus on additional rigorous studies to analyze the impact of nurse practitioner-delivered palliative care services specifically for HSCT patients. Projects will be necessary to evaluate models of care that directly integrate palliative care services onto inpatient hematology/HSCT units, working exclusively as a part of the standard care for patients. Additionally, future research could focus on the cost benefits of EPC for HSCT patient and the impact a partnership with palliative care has on the hematology/HSCT teams.

\section{CONCLUSION}

In conclusion, HSCT patients and their families undergo intensive treatments in search of a cure or long-term disease survival. These treatments can have devastating side effects, burdensome issues with fear and stress, and risk of death. The lack of EPC involvement for these patients continues to be an issue despite the demonstrated palliative care benefits in cancer care. The NEST Pathway was developed to create a partnership between palliative care services and HSCT teams. The pathway emphasizes teamwork and the necessity to rely on other professions to best care for patients and their families. Its use is necessary to make genuine strides toward facilitating the delivery of EPC for our HSCT patients, allowing quality of life to be the main driving goal.

palliative oncology care: Patient outcomes in the ENABLE III randomized controlled trial. Journal of Clinical Oncology, 33(13), 1438-1445. https://doi.org/10.1200/jco.2014.58.6362

Canadian Nurses Association. (2018). Nurse practitioner. https://cnaaiic.ca/en/professional-development/advanced-nursing-practice/ nurse-practitioners

Canadian Medical Association. (2015). Palliative care: Canadian medical association's national call to action. https://www.cma.ca/Assets/assetslibrary/document/en/advocacy/palliative-care-report-online-e.pdf 
Chung, H. M., Lyckholm, L. J., \& Smith, T. J. (2009). Palliative care in BMT. Bone Marrow Transplantation, 43(4), 265-273. https://doi. org/10.1038/bmt.2008.436

Clark, M. A., Ott, M., Rogers, M. L., Politi, M. C., Miller, S. C., Moynihan, L., Robison, K., Stuckey, A., \& Dizon, D. (2017). Advance care planning as a shared endeavor: Completion of ACP documents in a multidisciplinary cancer program. PsychoOncology, 26(1), 67-73. https://doi.org/10.1002/pon.4010

D’Ambruoso, S. F., Coscarelli, A., Hurvitz, S., Wenger, N., Coniglio, D., Donaldson, D., Pietras, C., \& Walling, A. M. (2016). Use of a shared mental model by a team composed of oncology, palliative care, and supportive care clinicians to facilitate shared decision making in a patient with advanced cancer. American Society of Clinical Oncology, 12(11), 1039-1045. https://doi.org/10.1200/ JOP.2016.013722

D’Souza, A., Lee, S., Zhu, X., \& Pasquini, M. (2017). Current use and trends in hematopoietic cell transplantation in the United States. Biology of Blood and Marrow Transplantation, 23(9), 1417-1421. https://doi.org/10.1016/j.bbmt.2017.05.035

Dans, M., Smith, T., Back, A., Baker, J. N., Bauman, J. R., Beck, A. C., Block, S., Campbell, T., Case, A. A., Dalal, S., Edwards, H., Fitch, T. R., Kapo, J., Kutner, J. S., Kvale, E., Miller, C., Misra, S., Mitchell, W., Portman, D. G., Spiegel, D., ... Scavone, J. L. (2017). NCCN guidelines insights: Palliative care, version 2.2017. The Official Journal of the National Comprehensive Cancer Network, 15(8), 989997. https://doi.org/10.6004/jnccn.2017.0132

Deitrick, L. M., Rockwell, E. H., Gratz, N., Davidson, C., Lukas, L., Stevens, D., Fitzgerald, G., Naugle, M., Wolf, J., \& Sikora, B. (2011). Delivering specialized palliative care in the community: A new role for nurse practitioners. Advances in Nursing Science, 34(4), 23-36. https://doi.org/10.1097/ANS.0b013e 318235834f

Dyar, S., Lesperance, M., Shannon, R., Sloan, J., \& Colon-Otero, G. (2012). A nurse practitioner directed intervention improves the quality of life of patients with metastatic cancer: Results of a randomized pilot study. Journal of Palliative Medicine, 15(8), 890895. https://doi.org/10. 1089/jpm.2012.0014

El-Jawahri, A. R., Traeger, L. N., Kuzmuk, K., Eusebio, J. R., Vandusen, H. B., Shin, J. A., Keenan, T., Gallagher, E. R., Greer, J. A., Pirl, W. F., Jackson, V. A., Ballen, K. K., Spitzer, T. R., Graubert, T. A., McAfee, S. L., Dey, B. R., Chen, Y. B., \& Temel, J. S. (2015). Quality of life and mood of patients and family caregivers during hospitalization for hematopoietic stem cell transplantation. Cancer, 121(6), 951-959. https://doi.org/10.1002/cncr.29149

El-Jawahri, A., LeBlanc, T., VanDusen, H., Traeger, L., Greer, J. A., Pirl, W. F., Jackson, V. A., Telles, J., Rhodes, A., Spitzer, T. R., McAfee, S., Chen, Y. A., Lee, S. S., \& Temel, J. S. (2016). Effect of inpatient palliative care on quality of life 2 weeks after hematopoietic stem cell transplantation: A randomized clinical trial. Journal of the American Medical Association, 316(20), 2094-2103. https://doi. org/10.1001/jama.2016.16786

Ferrell, B. R., Temel, J. S., Temin, S., Alesi, E. R., Balboni, T. A., Basch, E. M., Firn, J. I., Paice, J. A., Peppercorn, J. M., Phillips, T., Stovall, E. L., Zimmermann, C., \& Smith, T. J. (2017). Integration of palliative care into standard oncology care: American society of clinical oncology clinical practice guideline update. Journal of Clinical Oncology, 35(1), 96-112. https://doi.org/10.1200/ jco.2016.70.1474

Fox, K. (2014). The role of the acute care nurse practitioner in the implementation of the commission on cancer's standards on palliative care. Clinical Journal of Oncology Nursing, 18(Suppl), 39-44. https://doi.org/10.1188/14.CJON.S1.39-44
Harden, K. L., \& Schembri, J. A. (2016). Early intervention with transplant recipients to improve access to and knowledge of palliative care. Clinical Journal of Oncology Nursing, 20(4), E88-92. https://doi.org/10.1188/16.CJON

Ho, H. T., Barbera, L., Saskin, R., Lu, H., Neville, B. A., \& Earle, C. C. (2011). Trends in the aggressiveness of end-of-life cancer care in the universal health care system of Ontario, Canada. Journal of Clinical Oncology, 29(12), 1587-1591.

Hochman, M. J., Yu, Y., Wolf, S. P., Samsa, G. P., Kamal, A. H., \& LeBlanc, T. W. (2018). Comparing the palliative care needs of patients with hematologic and solid malignancies. Journal of Pain and Symptom Management, 55(1), 82-88. https://doi.org/10.1016/j. jpainsymman .2017 .08 .030

Howell, D. A., Shellens, R., Roman, E., Garry, A. C., Patmore, R., \& Howard, M. R. (2010). Hematological malignancy: Are patients appropriately referred for specialist palliative and hospice care? A systematic review and meta-analysis of published literature. Palliative Medicine, 25(6), 630-640. https://doi. org/10.1177/0269216310391692

Hui, D., Kim, S. H., Roquemore, J., Dev, R., Chisholm, G., \& Bruera, E. (2014). Impact of timing and setting of palliative care referral on quality of end-of-life care in cancer patients. Cancer, 120(11), 1743-1749.

Kain, D. A., \& Eisenhauer, E. A. (2016). Early integration of palliative care into standard oncology care: Evidenced and overcoming barriers to implementation. Current Oncology, 23(6), 374-377. https://doi.org/10.3747/co.23.3404

Kennedy, C., Brooks Young, P., Nicol, J., Campbell, K., \& Gray Brunton, C. (2015). Fluid role boundaries: Exploring the contribution of the advanced nurse practitioner to multiprofessional palliative care. Journal of Clinical Nursing, 24(21/22), 3296-3305. https://doi.org/10.1111/jocn.12950

LeBlanc, T. W., O'Donnell, J. D., Crowley-Matoka, M., Rabow, M. W., Smith, C. B., White, D. B., Tiver, G. A., Arnold, R. M., \& Schenker, Y. (2015). Perceptions of palliative care among hematologic malignancy specialists: A mixed-methods study. Journal of Oncology Practice, 11(2), e230-238. https://doi.org/10.1200/ JOP.2014.001859

Levine, D. R., Baker, J. N., Wolfe, J., Lehmann, L. E., \& Ullrich, C. (2017). Strange bedfellows no more: How integrated stem-cell transplantation and palliative care programs can together improve end-of-life care. Journal of Clinical Oncology, 13(9), 569-577. https:// doi.org/10.1200/JOP.2017.021451

Loggers, E. T., LeBlanc, T. W., El-Jawahri, A., Fihn, J., Bumpus, M., David, J., Horak, P., \& Lee, S. J. (2016). Pre-transplantation supportive and palliative care consultation for high-risk hematopoietic cell transplantation patients. Biology of Blood and Marrow Transplantation, 22(7), 1299-1305. https://doi.org/10.1016/j. bbmt.2016.03.006

May, P., Garrido, M. M., Cassel, J. B., Kelley, A. S., Meier, D. E., Normand, C., Smith, T. J., \& Morrison, R. S. (2017). Cost analysis of a prospective multi-site cohort study of palliative care consultation teams for adults with advanced cancer: Where do cost-savings come from? Palliative Medicine, 31(4), 378-386. https:// doi.org/10.1177/0269216317690098

Mitchell, G. K., Senior, H. E., Bibo, M. P., Makoni, B., Young, S. N., Rosenberg, J. P., \& Yates, P. (2016). Evaluation of a pilot of nurse practitioner led, GP supported rural palliative care provision. BMC Palliative Care, 15, 1-11. https://doi.org/10.1186/s12904-016-0163-y

Owens, D., Eby, K., Burson, S., Green, M., McGoodwin, W., \& Isaac, M. (2012). Primary palliative care clinic pilot project demonstrates benefits of a nurse practitioner-directed clinic 
providing primary and palliative care. Journal of the American Academy of Nurse Practitioners, 24(1), 52-58. https://doi. org/10.1111/j.1745-7599.2011.00664.x

O’Mahony, S., Johnson, T. J., Amer, S., McHugh, M. E., McHenry, J., Fosler, L., \& Kvetan, V. (2017). Integration of palliative care advanced practice nurses into intensive care unit teams. American Journal of Hospice and Palliative Medicine, 34(4). 330-334. https:// doi.org/10.1177/1049909115627425

Pfeil, T. A., Laryionava, K., Reiter-Theil, S., Hiddemann, W., Winkler, E. C. (2015). What keeps oncologists from addressing palliative care early on with incurable cancer patients? An active stance seems key. The Oncologist, 20(1), 56-61. https://doi.org/10.1634/ theoncologist.2014-0031

Roeland, E., \& Ku, G. (2015). Spanning the canyon between stem cell transplantation and palliative care. American Society of Hematology, 15, 484-489. https://doi.org/10.1182/ asheducation-2015.1.484

Selvaggi, K. J., Vick, J. B., Jessell, S. A., Lister, J., Abrahm, J. L., \& Bernacki, R. (2014). Bridging the gap: A palliative care consultation service in a hematological malignancy-bone marrow transplant unit. The Journal of Community and Supportive Oncology, 12(2), 50-55. https://doi.org/10.12788/jcso.0015

Smith, T. J., Temin, S., Alesi, E. R., Abernethy, A. P., Balboni, T. A., Basch, E. M., Ferrell, B. R., Loscalzo, M., Meier, D. E., Paice, J. A., Peppercorn, J. M., Somerfield, M., Stovall, E., \& Von Roenn, J. H. (2012). American Society of Clinical Oncology provisional clinical opinion: the integration of palliative care into standard oncology care. Journal of Clinical Oncology, 30(8), 880-887. https://doi. org/10.1200/JCO.2011.38.5161
Temel, J. S., Greer, J. A., Muzikansky, A., Gallagher, E. R., Admane, S., Jackson, V. A., Dahlin, C. M., Blinderman, C. D., Jacobsen, J., Pirl, W. F., Billings, J. A., \& Lynch, T. J. (2010). Early palliative care for patients with metastatic non-small-cell lung cancer. The New England Journal of Medicine, 363, 733-742. https://doi.org/10.1056/ NEJMoa1000678

Temel, J. S., Greer, J. A., Admane, S., Gallagher, E. R., Jackson, V. A., Lynch, T. J., Lennes, I. T., Dahlin, C. M., \& Pirl, W. F. (2011). Longitudinal perceptions of prognosis and goals of therapy in patients with metastatic non-small-cell lung cancer: Results of a randomized study of early palliative care. Journal of Clinical Oncology, 29(17), 2319-2326. https://doi.org/10.1200/ JCO.2010.32.4459

Walling, A. M., D'Ambruoso, S. F., Malin, J. L., Hurvitz, S., Zisser, A., Coscarelli, A., Clarke, R., Hackbarth, A., Pietras, C., Watts, F., Ferrell, B., Skootsky, S., \& Wenger, N. S. (2017). Effect and efficiency of an embedded palliative care nurse practitioner in an oncology clinic. Journal of Oncology Practice, 13(9), 792-799. https:// doi.org/10.1200/JOP.2017.020990

Zimmermann, C., Swami, N., Krzyzanowska, M., Hannon, B., Leighl, N., Oza, A., Moore, M., Rydall, A., Rodin, G., Tannock, I., Donner, A., \& Lo, C. (2014). Early palliative care for patients with advanced cancer: A cluster-randomized controlled trial The Lancet, 383(9930), 1721-1730. https://doi.org/10.1016/ S0140-6736(13)62416-2 